\title{
Avaliação dos idosos e a percepção dos profissionais quanto os riscos de quedas em uma instituição de longa permanência
}

\author{
Evaluation of elderly people and the perception of professionals \\ concerning the risk of falls in a long term care institution
}

Marilucia Vieira dos Santos, ${ }^{1}$ Simone Ely Weizenmann, ${ }^{1}$ Lydia Christmann Espindola Koetz. ${ }^{1}$ 'Centro Universitário UNIVATES, Lajeado, RS, Brasil.

Recebido em: dezembro 2014 / Aceito em: fevereiro 2015 bvieira78@bol.com.br

\section{RESUMO}

O processo de envelhecimento está relacionado com a diminuição do equilíbrio, dependência, medo de cair, institucionalização e quedas. Objetivo: avaliar o risco de quedas em idosos institucionalizados e identificar a percepção dos profissionais cuidadores quanto à prevenção de queda dos idosos na Instituição de Longa Permanência para Idosos (ILPI). Método: estudo de casos, descritivo e com abordagem quali-quantitativa. Participaram da pesquisa quatro idosos com 60 anos ou mais, institucionalizados, e quatro profissionais da saúde. Os dados foram coletados por meio de entrevista e aplicação da Escala de Equilíbrio de Berg com os idosos, análise ergonômica da ILPI e aplicação de um questionário com os profissionais. Resultados: o estudo mostrou que um idoso teve duas quedas no último ano na ILPI, e estes eventos restringiram a atividade de levantar-se sozinho por medo e insegurança, porém todos os idosos avaliados apresentaram risco de quedas. Ainda, verificou-se que a instituição não está adequada quanto aos aspectos ergonômicos, segundo as normas regulamentadoras. Os profissionais da ILPI apresentaram conhecimento sobre a saúde e cuidados com o idoso. Considerações finais: as alterações físicas dos idosos e as ergonômicas da ILPI avaliadas correspondem a risco de quedas. Apesar dos cuidadores apresentaram conhecimento sobre a saúde e prevenção de quedas do idoso e considerando importante a prática educativa, poucos profissionais demonstraram interesse em participar dos encontros propostos para apresentação e discussão dos resultados quanto ao risco de queda dos idosos.

Palavras-chave: Quedas; Idoso; Educação em Saúde; Fisioterapia.

\section{ABSTRACT}

The aging process is related to the decrease of balance, dependence, fear of falling, institutionalization and falls. Objective: evaluate the fall risk concerning institutionalized elderly people and identify the perception of professional caregivers in the prevention of falls in a Long-Term Care Facility (LTCF). Method: this is a descriptive field study of transverse nature and quali-quantitative approach. Four institutionalized 60-year-old (or over) elderly people took part in the survey, as well as four healthcare professionals. Data were collected through interview and application of Berg Balance Scale among the elderly people, ergonomic analysis of LTCF and a questionnaire with caregivers. Results: the study showed that an elderly subject had two falls in the last year at the LTCF and these events limited the act of stand up due to fear and insecurity, however $100 \%$ of the evaluated elderly subjects showed risk of falling. In addition, it was found that the institution is not adequate in ergonomic aspects according to regulatory standards. Caregivers from LTCF showed knowledge about elderly health and care. Final considerations: therefore the evaluated physical changes of elderly people and ergonomic changes of LTCF match to falls risk. Even though caregivers have knowledge about elderly health and falls prevention, considering the importance of educational practice, few professionals showed interest in participating of the proposed meetings in which were shown and discussed the results of elderly people's falling risks.

Keywords: Falls; Elderly people; Health Education; Physiotherapy. 


\section{INTRODUÇÃO}

O Rio Grande do Sul é o quarto Estado brasileiro em número absoluto de idosos e o segundo em expectativa de vida, com média de 75,5 anos para ambos os sexos, conforme dados do IBGE'1. Esses dados mostram a necessidade em criar ações capazes de oferecer respostas às necessidades dos idosos e de suas famílias.

A expectativa de vida ao nascer no Brasil em 2010 foi de 73,4 anos para ambos os sexos. Para os homens, a expectativa de vida era de 69,73 anos e de 77,32 anos para as mulheres, o que representa uma diferença de 7,5 anos de vida a mais para as mulheres no país ${ }^{1}$. 0 Estado do Rio Grande do Sul, cuja população total é de 10.693.929, possui 1.459.597 idosos, o que representa $13,65 \%$ de sua população.

Nos últimos anos, tem ocorrido um aumento nas taxas de idosos institucionalizados. No Brasil, existem 200 mil abrigos para idosos, e a maior parte de residentes é do sexo feminino ${ }^{2}$. O idoso passou a liderar o crescimento populacional, como reflexo da queda da fecundidade e da mortalidade no país nos anos $60^{3}$.

A razão do crescente envelhecimento populacional e das dificuldades que a família encontra para o desempenho das funções a ela atribuídas como cuidadora, gera o aumento da demanda por serviços e instituições para o atendimento e acompanhamento integral ao idoso ${ }^{4}$.

Diante do novo perfil epidemiológico, surge a necessidade de prevenção e assistência aos pacientes idosos, através de programas que incluam equipes interdisciplinares, buscando conhecimento sobre o envelhecimento, priorizando a manutenção da independência e autonomia do idoso, para desempenhar as atividades básicas de vida diária e prolongar a capacidade funcional ${ }^{5-7}$.

A saúde dos idosos sofrem influências de vários fatores, no entanto, avaliar e promover a saúde do idoso significa considerar distintos campos do saber, em uma atuação interdisciplinar ${ }^{8}$. Dessa forma, a avaliação funcional é fundamental e deve ser incluída a qualquer consulta ou entrevista pelos profissionais de saúde, pois ela identifica as limitações e as potencialidades, possibilitando ao profissional de saúde traçar um plano de intervenção com medidas preventivas, terapêuticas e reabilitadoras ${ }^{6}$.

Sabe-se que o processo de envelhecimento envolve várias alterações em todos os sistemas do corpo humano; independente da causa, as alterações aumentam a instabilidade, alteram o padrão de marcha e podem predispor a perda ou diminuição da capacidade funcional; consequentemente aumentando o risco de quedas. Portanto, surge a necessidade da preparação e adequação dos serviços de saúde, incluindo a formação e capacitação de profissionais para o atendimento desta demanda ${ }^{9,10}$.

Deve-se considerar também que a análise ergonômica do ambiente de uma instituição de longa permanência para idosos objetiva conhecer os fatores de risco existentes que podem vir a comprometer a independência dos idosos e ocasionar quedas. Na busca por saúde preventiva é de extrema importância que, para o planejamento das ações, os fatores físico corporal, ambiental e recursos humanos sejam avaliados, para que a educação em saúde seja efetiva ${ }^{9}$.

Segundo o Estatuto do Idoso (Lein $\left.{ }^{\circ} 10.741 / 2003\right)^{11}$ em seu Artigo 18, do direito à saúde, diz: "As instituições de saúde devem atender às necessidades do idoso, promovendo o treinamento e a capacitação dos profissionais, assim como orientação a cuidadores familiares e grupos de auto ajuda"12.

Diante desta situação a pesquisa teve como objetivo avaliar o risco de quedas em idosos institucionalizados e identificar a percepção dos profissionais cuidadores, quanto à prevenção de queda dos idosos na Instituição de Longa Permanência para Idosos (ILPI).

\section{METODOS}

Abordagem metodológica, caracterização do local e amostra A metodologia é de estudo de casos, descritivos com abordagem quali-quantitativa. O presente estudo ocorreu em uma ILPI, no município de Lajeado/RS, no período de agosto a outubro de 2014.

Para a realização do estudo foram incluídos idosos, com idade igual ou superior a 60 anos, sendo que dos 15 idosos da ILPI, 04 participaram e o restante dos idosos foram excluídos por apresentarem alguma incapacidade de compreensão ao comando verbal independente do tipo de patologia, por serem cadeirantes ou estarem acamados.

Também participaram da pesquisa os profissionais da saúde que atuam no cuidado direto com o idoso. Dos 07 profissionais que trabalham na instituição participaram 04, sendo excluídos os auxiliares de serviços gerais e os que não estavam presentes no momento dos encontros. Os profissionais tinham como formação auxiliar e técnico de enfermagem, ensino superior incompleto de Enfermagem e graduado em enfermagem. E estes, apresentavam de 5 a 7 meses de tempo de serviço na ILPI.

Todos os voluntários da amostra assinaram o termo de consentimento livre e esclarecido. A pesquisa foi aprovada pelo Comitê de Ética em Pesquisa em Seres Humanos do Centro Universitário UNIVATES, sob o protocolo $\mathrm{n}^{\circ} 724.135 / 2014$. Apresentando os critérios éticos de acordo com a resolução 466/12 do Conselho Nacional de Saúde

\section{Procedimentos Metodológicos}

Primeiramente, foi realizada uma entrevista com os idosos que contemplava dados como idade, sexo, tempo na ILPI, diagnóstico médico e questões relacionadas às quedas. Após, foi utilizada a Escala de Equilíbrio de Berg (EEB), instrumento validado, de avaliação funcional do equilíbrio estático e dinâmico, composta de 14 tarefas, pontuando de 0-4 para cada uma, sendo que: 0 - é incapaz de realizar a tarefa e 4 - realiza a tarefa de forma independente. $O$ escore total varia de 0 - 56 pontos $^{14,15}$. Quando o escore for abaixo de 45 pontos, considera-se risco de queda. As perguntas correspondentes ao teste foram realizadas pelo pesquisador, acompanhando o roteiro da Escala de Equilíbrio de Berg. 0 teste foi realizado próximo de uma cadeira; se necessário, o idoso senta para descansar, evitando a queda.

Após, foi realizada uma análise ergonômica dos ambientes para reconhecimento dos espaços, com a finalidade de avaliar para orientar a elaboração de am- 
bientes mais seguros e adequados e proporcionar bem estar, conforto e qualidade de vida para os idosos. Para essa avaliação foi utilizado um questionário Check Listanálise ergonômica, que teve como base na portaria $\mathrm{n}^{\circ}$ 810/8916 do Ministério da Saúde e da NBR 9050 ${ }^{17}$, na qual estão descritas as normas e padrões para o funcionamento de casas de repouso, clínicas geriátricas e outras instituições destinadas ao atendimento de idosos, quanto à área física.

Em um segundo momento, foi aplicado um questionário com os funcionários da ILPI, contendo 6 questões abertas e descritivas, com o objetivo de avaliar e verificar o nível de conhecimento relacionado às alterações fisiológicas dos idosos, fatores que influenciam nas quedas, cuidados ergonômicos e qual o entendimento e importância da Educação Continuada. Além disso, foram realizados mais dois encontros, com objetivo de informar os profissionais à respeito dos resultados das análises da Escala de Equilíbrio de Berg e da avaliação ergonômica da ILPI. Ainda, considerando estas análises realizadas, os profissionais foram informados sobre alterações fisiológicas decorrentes do processo de enveIhecimento e receberam dicas de medidas preventivas e cuidados ergonômicos para evitar as quedas dos idosos dentro de uma ILPI, através de material impresso.

Os resultados dos dados quantitativos foram tabulados, analisados através do programa Excel. Após, foram feitas correlações estatísticas descritivas, considerando o valor de médio e desvio padrão. Para análise do EEB foi considerado os valores absolutos da escala. Para os dados qualitativos foram estabelecidos as seguintes categorias de análises: a) Análise ergonômica da ILPI e b) A percepção dos profissionais cuidadores na prevenção de risco de queda do idoso institucionalizado.

\section{RESULTADOS}

A tabela 1 apresenta a caracterização da amostra dos idosos, que foi composta por 4 indivíduos, cuja idade variou de 73 a 87 anos, com idade média de 79 anos $( \pm 4,8)$, sendo 2 do sexo feminino e 2 do sexo masculino. O tempo de institucionalização variou de 4 a 7 meses, com tempo médio de 5,5 meses ( $D P \pm 1,1)$.

No histórico de quedas, o idoso $D$ teve dois episódios no último ano, sendo que ocorreram no refeitório da ILPI, durante o dia, devido à hipotensão arterial, consequentemente apresentando escoriações na cabeça. Devido a estes episódios, o sujeito restringiu a atividade de levantar-se sozinho por medo e insegurança.

$\mathrm{Na}$ Escala de Equilíbrio de Berg (EEB), verificou-se que os idosos A, B, C e D apresentaram pontuação igual ou menor de 36 , indicando $100 \%$ de risco de quedas (Tabela 2).
Tabela 2 - Escala de Equilíbrio de Berg (EEB).

\begin{tabular}{lcccc}
\hline Idoso & A & B & C & D \\
\hline Pontuação Total & 33 & 36 & 34 & 34 \\
\hline
\end{tabular}

A tabela 3 mostra todos os itens da Escala de Equilíbrio de Berg, verificando que todos os idosos avaliados tiveram maior dificuldade em realizar tarefas que exigissem equilíbrio dinâmico, expressados pelas tarefas 9, 10, 11, 13 e 14 (Tabela 3).

Tabela 2 - Escala de Equilíbrio de Berg (EEB).

\begin{tabular}{|c|c|c|c|c|c|}
\hline Questões & 0 & 1 & 2 & 3 & 4 \\
\hline 1.Sentado para em pé & & & & 4 & \\
\hline 2.Em pé sem suporte & & & & & 4 \\
\hline 3.Sentado com as costas sem suporte mas os & & & & & 4 \\
\hline \multicolumn{6}{|l|}{ pés apoiados no chão ou sobre um banquinho } \\
\hline 4.De pé para sentado & & & & 3 & \\
\hline (Continuação da tabela 3) & & & 1 & & \\
\hline 5. Transferências & & & & 4 & \\
\hline 6.Em pé sem apoio com os olhos fechados & & 1 & & & 3 \\
\hline 7.Em pé sem apoio com os pés unidos & & 1 & & & 3 \\
\hline 8. Estendendo o braço à frente enquanto está em pé & & & 2 & 1 & 1 \\
\hline 9.Apanhar um objeto do chão estando em pé & 2 & 1 & & 1 & \\
\hline 10. Virando e olhando para trás por cima do & & & 3 & & 1 \\
\hline \multicolumn{6}{|l|}{ ombro esquerdo e direito estando em pé } \\
\hline 11. Girando 360 graus & 1 & & 2 & & 1 \\
\hline 12.Colocando pés alternados sobre um degrau & 1 & & & 3 & \\
\hline \multicolumn{6}{|l|}{ ou banquinho estando em pé sem apoio } \\
\hline 13.Em pé sem suporte com um pé na frente & 3 & 1 & & & \\
\hline 14.Em pé sobre uma perna & 3 & 1 & & & \\
\hline
\end{tabular}

\section{Análise ergonômica da ILPI}

$\mathrm{Na}$ análise ergonômica da ILPI, observou-se que a construção é horizontal, com saídas de emergência sinalizadas. A casa não possui escadas, nem portas de contenção. O chão não está encerado e não há tapetes, como recomendado. A residência possui iluminação natural por portas e janelas e iluminação artificial com lâmpadas fluorescentes, porém não possui luz de vigília nos dormitórios e banheiros. As portas internas não possuem vão luz de $0,80 \mathrm{~m}$ como recomendado, porém as portas de correr estão de acordo, possuindo trilhos fixos na parte superior com as maçanetas das portas do tipo alavanca instaladas a $1,10 \mathrm{~m}$.

Nos espaços de convivência as cores das paredes são variadas; nos quartos, há presença de cores como verde claro, azul, rosa, vermelho, na sala de TV os tons são amadeirados foscos e nas demais dependências as cores são pálidas e não possuem brilho.

Nos quartos, 1, 4, 5 e 6, alguns dos leitos não apresentam 1,00m de distância entre eles. Todos os leitos

Tabela 1 - Caracterização da amostra dos idosos.

\begin{tabular}{lccccc}
\hline Idoso & A & B & C & D & Valor Médio \\
\hline Idade & 83 anos & 87 anos & 73 anos & 77 anos & DP \\
Sexo & Feminino & Feminino & Masculino & Masculino & - \\
Tempo de Instituição & 5 meses & 6 meses & 4 meses & 7 meses & 5,5 \\
Diagnóstico médico & Alzheimer & Cardiopatias e & AVE & Diagnóstico & - \\
& & problemas respiratórios & & Indefinido \\
\hline
\end{tabular}


próximos da parede não respeitam a distância paralela de $0,50 \mathrm{~m}$. Os quartos 4, 5 e 6 não apresentam área mínima de $5 \mathrm{~m}^{2}$ por leito e o quarto 2 não possui área mínima de $6,5 \mathrm{~m}^{2}$, quando um leito por quarto. Nos 6 quartos avaliados não há luz presente (abajur), não tem campainha na cabeceira dos leitos e não há grades nas camas.

As poltronas da sala são macias e possuem braços. No refeitório, as mesas são de material MDF, possuem 4 pés e $82 \mathrm{~cm}$ de altura; as cadeiras são de madeira com 4 pés e sem braços de apoio. No acesso entre o corredor e a varanda há uma rampa com 1,45m de largura, sem piso antiderrapante e sem corrimão, que não está em conformidade. O corredor principal que dá acesso aos quartos e banheiros não está adequado, possuindo largura de 1,20m, apresentando corrimão apenas de um lado com $5 \mathrm{~cm}$ de diâmetro, instalado a $90 \mathrm{~cm}$ do chão e $2 \mathrm{~cm}$ da parede.

Os sanitários não são separados por sexo, o vaso não está instalado sobre um sóculo de $0,15 \mathrm{~m}$ de altura e não possui barras de apoio nem botão de campainha. Porém, possui 01 vaso na proporção para cada 6 pessoas, como recomendado. Todas as portas dos sanitários abrem para dentro e não estão instaladas a $0,20 \mathrm{~m}$ na parte inferior.

\section{A percepção dos profissionais cuidadores na prevenção} de risco de queda do idoso institucionalizado

Após a análise dos questionários realizados com os profissionais cuidadores da ILPI, os mesmos relataram sobre as alterações decorrentes do processo de enveIhecimento. Citaram que as alterações ocorrem na parte digestiva, além de limitações físicas como banhar-se, vestir-se, deambular, perda cognitiva, sintomas depressivos, isolamento social, declínio sensorial, quedas, dificuldade para manter o controle das necessidades fisiológicas e alterações na marcha. Também apontaram que os fatores que influenciam nas quedas são a osteopenia, perda da mobilidade funcional, a insegurança para deambular, alterações de visão e vertigem, fatores relacionados ao ambiente ergonômico (tapetes, piso escorregadio, banheiros não adaptáveis), alterações de postura e equilíbrio com consequente alteração de marcha.

Referente aos cuidados ergonômicos foi possível perceber que os profissionais sabem da importância e citam algumas medidas ergonômicas que são fundamentais, como corrimões, barras de apoio, pega-mão, pisos antiderrapantes. Para transferência dos idosos, auxiliam informando sobre o que será feito e para onde estão sendo transferidos; ofertam pontos de apoio e solicitam ao idoso para colaborar sempre que possível, e quando necessário solicitam ajuda de mais um profissional para auxiliar na transferência para evitar o risco de quedas.

Os cuidadores da ILPI consideraram importante a educação continuada em saúde e a consideram como uma forma de aperfeiçoamento, de busca de novos conhecimentos e novas práticas de saúde, que têm como objetivo melhorar na qualidade do atendimento, tornando-o eficiente, planejado e contínuo. Ressaltam que um grupo de educação continuada é de grande importância dentro de uma ILPI e de qualquer outra instituição de saúde.

\section{DISCUSSÃO}

O aumento da expectativa de vida nos países menos desenvolvidos, como o Brasil, tem sido evidenciada pelos avanços tecnológicos relacionados à área de saúde nos últimos $60 \operatorname{anos}^{18}$. A presente pesquisa mostrou que os idosos apresentavam idade média de 79 anos (DP \pm 4,8). Conforme estudo de Martins e colaboradores $^{12}$ destaca-se o crescimento da população idosa no Brasil e também, a necessidade de estudos para compreendê-la, com vistas a implantar políticas de saúde específicas.

Neste estudo o participante $D$ teve episódios de quedas e todos os idosos avaliados apresentaram 100\% de risco de quedas. Sabe-se que o processo de enveIhecimento está relacionado com a diminuição do equilíbrio, dependência, medo de cair, institucionalização, e quedas ${ }^{19}$. Sendo que este último fator é identificado como um grave problema de saúde pública, devido à frequência, morbidade e elevado custo social e econômico decorrente das lesões provocadas. As alterações do equilíbrio e da marcha também podem estar associadas ao medo de cair, levando a restrições da mobilidade e insegurança entre os idosos, favorecendo assim a ocorrência de quedas ${ }^{20}$. O envelhecimento provoca a perda progressiva da capacidade de adaptação ao meio em que vive, privando as pessoas idosas dos indícios sensoriais necessários para perceber o ambiente e se relacionar com ele ${ }^{9}$.

As causas de quedas podem estar relacionadas a fatores intrínsecos, como alterações fisiológicas próprias do envelhecimento, as condições patológicas e efeitos adversos a medicações; e fatores extrínsecos, como um corredor mal iluminado e tapetes escorregadios ${ }^{21,22}$.

A internação em instituições de longa permanência para idosos (ILPI) também pode ser considerado um fator de risco para quedas, sendo necessário atenção e cuidados especiais; porém, a maioria das instituições encontra-se desprovida de adaptações ou quando possui, são introduzidas de maneira inadequada ${ }^{9-23}$.

\section{Análise ergonômica da ILPI}

$\mathrm{Na}$ análise ergonômica do estudo foi possível verificar que a edificação está na horizontal, o que facilita a locomoção e evita acidentes como quedas. Estes dados corroboram com as normas do Estatuto do Idoso de 2003, que diz se caso a edificação possuírem mais de um plano e não dispuserem de equipamentos adequados como rampa ou elevador para a circulação vertical, estas instituições só poderão atender pessoas imobilizadas no leito e com problemas locomotores ou psíquicos, no pavimento térreo. A ILPI estudada não possui escadas, nem portas de contenção, o que facilita ainda mais a deambulação e o deslocamento dos idosos. Por outro lado, as portas internas não possuem vão luz de no mínimo 0,80m; conforme rege a Portaria $n^{\circ}$ 810/89, dificultando a passagem de cadeira de rodas.

O corredor principal que dá acesso aos quartos e banheiros não está adequado, divergindo com a Portaria $n^{\circ} 810 / 89$ que diz que os corredores principais das instituições deverão ter largura mínima de 1,50m, com 
corrimão em ambos os lados, instalados a $0,80 \mathrm{~m}$ do piso e distantes $0,05 \mathrm{~m}$ da parede. E segundo a NBR 9050 (2004), os corrimãos devem ter o diâmetro entre $3,0 \mathrm{~cm}$ e $4,5 \mathrm{~cm}$, devidamente fixas às paredes com um espaço livre de no mínimo $4,0 \mathrm{~cm}$ entre a parede e a barra ${ }^{16,17}$.

A rampa de acesso entre o corredor da sala para a varanda não está de acordo com a Portaria $\mathrm{n}^{\circ}$ 810/89, que afirma que o piso deve ser revestido com material não derrapante, permitindo o livre rolamento de cadeiras de rodas.

As paredes dos quartos apresentavam tom colorido. Porém, as cores utilizadas não estavam adequadas, como o rosa que é considerado uma cor pálida, e o verde e o azul que são cores frias. Sugere-se que as paredes sejam coloridas e estimulantes ${ }^{24}$, sendo que as cores "quentes" em tons fortes são mais estimulantes; as frias, tais como azul e verde, apesar de serem ideais para reduzir tensão e estresse e oportunizar tranquilidade e calma no ambiente, não são visualizadas pelos idosos. Os tons pastéis, de lavandas e rosa podem parecer tão similares que poderiam confundir o idoso, na distinção entre seu próprio quarto e o do vizinho ${ }^{25}$.

Os quartos, 1, 4, 5 e 6 não estão de encontro com a Portaria $n^{\circ} 810 / 89$, pois os dormitórios devem possuir medida linear mínima de 2,5m. Da mesma forma os resultados mostram que a distância entre os leitos não estão de acordo com a norma. Em um estudo realizado em uma ILPI em 2010, que analisa os riscos de queda em uma IPLI, verifica que os locais onde mais ocorreram quedas foram: em primeiro lugar, a rua $(30,9 \%)$, seguido do quarto (25\%) e após do banheiro $(17,6 \%)$, sendo que o turno de maior ocorrência foi o diurno $(85,8 \%)^{26}$.

De acordo com a literatura, as poltronas e cadeiras não devem ser muito baixas, macias ou fundas, devido ao idoso apresentar maior dificuldade em sentar e levantar ${ }^{24}$. Segundo a NBR 9050, as cadeiras do refeitório também devem possuir braços de apoio e serem fixas no chão ${ }^{17,25}$.

No presente estudo os perigos ambientais encontrados na ILPI correspondem a riscos de quedas e percebe-se que algumas adaptações estão de acordo e outras necessitam serem revistas e adaptadas com enfoque ergonômico direcionado no cuidado com os idosos.

A maioria das ILPI no Brasil são filantrópicas, oferecendo cama e comida, sem infraestrutura adequada e sem profissionais habilitados para cuidar dos idosos. Tal situação reflete a ausência de conhecimento ou fiscalização da implementação das políticas públicas e de planejamento na (des) assistência prestada ${ }^{27}, 28$. Na presente pesquisa também evidenciou-se a necessidade de adequação ergonômica e dos recursos humanos, para garantir a saúde plena do idoso, que apesar de ser um estudo de um de uma única ILPI, as demandas encontradas reforçam a realidade de muitos lares para idosos.

\section{A percepção dos profissionais cuidadores na prevenção} de risco de queda do idoso institucionalizado

Os profissionais da saúde da pesquisa se autoavaliaram com um conhecimento bom sobre as alterações fisiológicas decorrentes do processo de envelhecimento; porém, a maioria dos profissionais possui pouca experiência prática com idosos, considerando o tempo de serviço.

A falta de conhecimento para a realização do trabalho pode resultar em experiências não satisfatórias, com consequentes déficits na prestação de cuidado ao idoso ${ }^{29}$. O Ministério da Saúde, em 1999, instituiu a Política de Saúde dos Idosos, por meio da Portaria n. ${ }^{\circ}$ 2.528/GM, e entre os temas abordados na política nacional para esta faixa etária, está a promoção do envelhecimento saudável e a manutenção da capacidade funcional, pelo maior tempo possível ${ }^{30}$.

Sabe-se que uma das alternativas importantes para assegurar a autonomia e independência do idoso, como também o envelhecer saudável, é a ação educativa, pois é um processo dinâmico que deve estimular o diálogo, indagação, reflexão, e ação partilhada, com objetivo de capacitar os profissionais envolvidos em busca da melhoria das condições de saúde da população idosa ${ }^{12}$. A partir do conhecimento das necessidades destes profissionais é possível criar ações educativas em saúde, contribuindo na melhoria da assistência prestada ${ }^{31}$.

A educação é uma estratégia para construir a capacidade de políticas e práticas efetivas de prevenção de quedas, para os profissionais de saúde envolvidos e para os responsáveis pelo design e pela construção de habitações e espaços públicos usados pelos idosos ${ }^{32}$.

As necessidades de capacitação são apontadas a partir da problematização do processo e da qualidade do trabalho nos serviços de saúde, facilitando a aplicação de conteúdos e tecnologias apropriadas ${ }^{13}$. A presente pesquisa contribuiu para este processo, mostrando que a ILPI apresenta fatores de risco para quedas dos idosos, tanto por inadequações arquitetônicas, quanto por alterações físicas fisiológicas decorrentes do envelhecimento. Além disso, os profissionais da saúde participante do estudo reconhecem a importância do aperfeiçoamento profissional constante para melhor atender os idosos institucionalizados.

Segundo a literatura, a excelência da prestação de cuidados aos idosos envolve a participação de todos os profissionais, através da abordagem cooperativa e global na prestação de cuidados $^{29}$. Os profissionais da ILPI estudada consideraram importante a prática educativa, porém poucos demonstraram interesse em participar dos encontros propostos para apresentação e discussão dos resultados encontrados nas análises realizadas quanto ao risco de queda dos idosos. Dessa forma, observa-se uma contradição entre o relato dos cuidadores considerando importante a educação continuada e atitude de não participação na discussão sobre a necessidades da ILPI assegurar condições de saúde plena, minimizando assim o risco de queda dos idosos.

A análise desta pesquisa faz refletir na atual situação social da pessoa idosa no Brasil, revelando a necessidade de discussões mais profundas sobre a saúde dos idosos, aspecto que deve ser enfatizado nas salas de aula $^{18}$, sobretudo na formação de profissionais da área de saúde ${ }^{33}$ para que estes sejam críticos e capazes de efetivar práticas inovadoras, em especial na prevenção que de quedas do idoso. 


\section{CONSIDERAÇÕES FINAIS}

Verificou-se que as alterações físicas dos idosos e as ergonômicas da ILPI correspondem a risco de quedas. Apesar dos cuidadores apresentaram conhecimento sobre a saúde e prevenção de quedas do idoso, considerando importante a prática educativa, poucos profissionais demonstraram interesse em participar dos encontros propostos para apresentação e discussão dos resultados da pesquisa. Sendo assim, sugerem-se novos estudos para verificar os efeitos das práticas de ações educativas após análise dos riscos de quedas em mais de uma ILPI.

\section{REFERÊNCIAS}

1. IBGE. Instituto Brasileiro de Geografia e Estatística. Primeiros resultados definitivos do Censo 2010: População do Brasil é de 190.755.799 pessoas [Internet] 2011 [Acesso em 2014 abr 12]. Disponível em: <www.ibge.gov.br/home/estatistica/ populacao/censo 2010/defaultt_sinopse.shtm>.

2. Pavan FJ, Meneghel SN, Junges JR. Mulheres idosas enfrentando a institucionalização. Cad Saúde Pública 2008 Set; 24(9): 2187-2189.

3. Depolito C, Leocadio PLLF, Cordeiro RC. Declínio funcional de idosa institucionalizada: aplicabilidade do modelo da Classificação Internacional de Funcionalidade, Incapacidade e Saúde. Fisioter Pesqui 2009 jun; 16(2): 183-189.

4. Ferreira OGL, et al. Significados atribuídos ao envelhecimento: idoso, velho e idoso ativo. Psico-USF 2010 dez; 15(3): 357-364.

5. Cruvinel TAC. Promoção da saúde e qualidade de vida dos idosos na saúde da família. [Monografia]. Uberaba: Curso de Pós Graduação Em Atenção Básica em Saúde da Família da Universidade Federal de Minas Gerais; 2005.

6. Nakatani AYK, et al. Capacidade funcional em idosos na comunidade e propostas de intervenções pela equipe de saúde. Rev Eletr Enf, 2009; 11(1): 144-150.

7. Guimarães LHCT, et al. Avaliação da capacidade funcional de idosos em tratamento fisioterapêutico. Rev Neurocienc, 2004 set; 12(3):130-133.

8. Ciosak $\mathrm{SI}$, et al. Senescência e senilidade: novo paradigma na atenção básica de saúde. Rev Esc Enferm USP, 2011 dez; 45(spe2):1763-1768.

9. Dagostim DK. Análise dos fatores ambientais de uma instituição de longa permanência para idosos. [Monografia]. Criciúma: Curso de Fisioterapia da Universidade do Extremo Sul Catarinense UNESC; 2009.

10. Melo MM. Prevenção de acidentes domésticos em idosos: relato de Experiência. V Simpósio de Ensino de Graduação [Internet]. 2007 p. 1 a 42007 [Acesso em 2014 abr 12] p.1-4. Disponível em: <http://www.unimep.br/phpg/ mostraacademica/anais/5mostra /4/240.pdf $>$.

11. BRASIL, Lei $n^{\circ} 10.741 / 2003$. Dispõe sobre o Estatuto do Idoso e dá outras providências. [Acesso em $2014 \mathrm{dez} 16]$ Disponível em: <http://www.planalto.gov.br/ccivil_03/ leis/2003/l10.741.htm>.

12. Martins JJ, et al. Necessidades de educação em saúde dos cuidadores de pessoas idosas no domicílio. Texto ContextoEnferm 2007 jun; 16(2): 254-262.

13. Silva MF, Conceição FA, Leite MMJ. Educação continuada: um levantamento de necessidades da equipe de enfermagem. Arq Bras Ciên Saúde 2009 abr; 34(1): 15-21.

14. Gazzola JM, et al. Fatores associados ao equilíbrio funcional em idosos com disfunção vestibular crônica. Rev Bras Otorrinolaringol 2006 out; 72(5):683-690.
15. Christofoletti G, et al. Risco de quedas em idosos com doença de Parkinson e demência de Alzheimer: um estudo transversal. Rev Bras Fisioter 2006 dez; 10(4):429-433.

16. Brasil. Ministério da Saúde. Portaria $n^{\circ}$. 810, de 22 de setembro de 1989. Normas para funcionamento de casas de repouso, clínicas geriátricas e outras instituições destinadas ao atendimento de idosos. Diário Oficial União 27 set 1989. Seção 1: 11297-17298.

17. Associação Brasileira de Normas Técnicas. NBR 9050: Acessibilidade a edificações, mobiliário, espaços e equipamentos urbanos. Rio de Janeiro: ABNT; 2004.

18. Mendes MRSSB, et al. A situação social do idoso no Brasil: uma breve consideração. Acta Paul Enferm 2005 dez; 18 (4) $422-426$.

19. Alves NB, Scheicher ME. Equilíbrio postural e risco para queda em idosos da cidade de Garça, SP. Rev Bras Geriatr Gerontol 2011 dez; 14(4): 763-768.

20. Borges LL, Garcia PA, Ribeiro SOV. Características clínicodemográficas, quedas e equilíbrio funcional de idosos institucionalizados e comunitários. Fisioter Mov 2009 mar; 22(1): 53-60.

21. Chaimowicz F, Barcelos EM, Madureira MDS, Ribeiro MTF. Saúde do idoso. Belo Horizonte: Coopmed; 2009.

22. Menezes RL, Bachion MM. Estudo da presença de fatores de riscos intrínsecos para quedas, em idosos institucionalizados. Ciênc Saúde Coletiva 2008 ago; 13(4): 1209-1218.

23. Santos MLC, Andrade MC. Incidência de quedas relacionadas aos fatores de riscos em idosos institucionalizados. Rev Baiana Saúde Pública 2005 jun; 29(1): 57-68.

24. Papaléo Netto M. Gerontologia: A velhice e o envelhecimento em visão globalizada. São Paulo: Atheneu; 2002.

25. Pascale MA. Ergonomia e alzheimer: a contribuição dos fatores ambientais como recurso terapêutico nos cuidados de idosos portadores da Demência do tipo Alzheimer. [Dissertação]. Florianópolis: Mestrado em Engenharia de Produção da Universidade Federal de Santa Catarina; 2002.

26. Álvares LM, Lima RC, Silva RA. Ocorrência de quedas em idosos residentes em instituições de longa permanência em Pelotas, Rio Grande do Sul, Brasil. Cad. Saúde Pública, 2010 jan, 26(1):31-40.

27. Mendonça JA, Marques Neto JF. Qualidade de vida do idoso institucionalizado frente aos grupos de afecções crônicas. Rev Ciênc Méd 2003 dez; 12(4): 299-306.

28. Silva $\mathrm{BCO}$, et al. Experiências vivenciadas por acadêmicos de enfermagem com idosos institucionalizados e não institucionalizados. Rev Kairós Gerontol 2012 jun; 15(3): 179-189.

29. Bissoli PGM, Cachioni M. Educação gerontológica: breve intervenção em centro de convivência-dia e seus impactos nos profissionais. Rev Kairós Gerontologia 2011 set; 14(4):143-164.

30. Brasil. Ministério da Saúde. Portaria $n^{\circ}$. 2.528, de 19 de outubro de 2006. Aprova a Política Nacional de Saúde da Pessoa Idosa. Diário Oficial União 20 out 2006; Seção 1: 142.

31. Carli R, Winck MT, Colomé ICS, Resta DG, Jahn AC. $\mathrm{O}$ trabalho de cuidadores de idosos institucionalizados: subsídios para a qualificação da assistência à saúde do idoso. Cienc Cuid Saúde 2008; 7(supl 2).

32. Organização Mundial de Saúde (OMS). Envelhecimento ativo: uma política de saúde. Brasília: Organização Pan-Americana de Saúde; 2005.

33. Brasil. Ministério da Saúde. Secretaria de Gestão do Trabalho e da Educação na Saúde. Políticas de educação e desenvolvimento para o SUS: caminhos para a educação permanente em saúde. Brasília: Ministério da Saúde; 2004. 